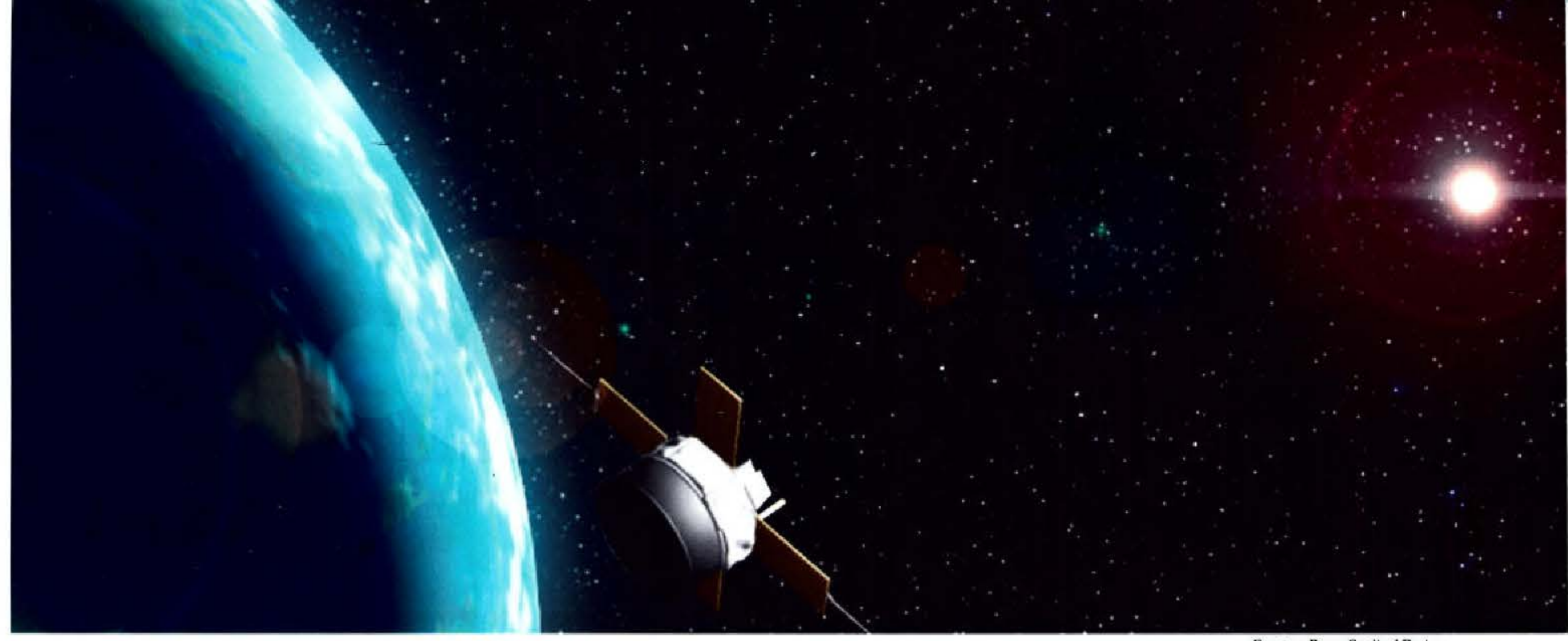

\title{
Solar and Interplanetary Data From the Advanced Composition Explorer
}

\author{
New data products from NASA's Advanced \\ Composition Explorer during its extended mission
}

\author{
By A.J. Davis, R.A. Mewaldt, E.C. Stone, and Charles W. Smith
}

ince early in 1998, NASA's Advanced Composition Explorer (ACE) spacecraft has provided continuous measurements of solar wind and energetic particle activity from L1, its orbit position located approximately $0.01 \mathrm{AU}$ sunward of Earth. Its mission addresses a wide range of objectives, including studying the origin and evolution of solar, interplanetary, and galactic material, the formation of the solar corona, the acceleration of the solar wind and coronal mass ejections, and the acceleration of energetic particles. In this article we review current and planned data products available from ACE and describe recent modifications to the spacecraft's orbit that will extend its lifetime and provide solar wind data closer to the EarthSun line.

The instruments on ACE measure ions and electrons accelerated at the Sun, in interplanetary space, at the edge of the heliosphere, and in the galaxy. The craft's six high-resolution spectrometers and three monitoring instruments characterize the environment by measuring the elemental, isotopic, and ionic charge-state composition of energetic nuclei at energies ranging from $\sim 1 \mathrm{keV} /$ nucleon in the solar wind to $\sim 0.5$ $\mathrm{GeV} /$ nucleon in cosmic radiation, as well as electrons from solar wind energies up to $\sim 300 \mathrm{keV}$ and magnetic field parameters. In addition, ACE has made key contributions to the rapidly growing field of space weather by providing con- tinuous measurements of solar wind conditions upstream of Earth that are freely available on the Internet. The spacecraft and instruments are described in detail in a 1998 paper by Stone et al. published in Space Science Reviews, and a series of accompanying papers.

Following the NASA Senior Review in 2003, ACE was approved for a multiyear extended mission. With this milestone comes the need for new data products and a modified trajectory to better serve the long-term needs of the space science community.

\section{ACE Data Available to the Scientific Community}

There are several types of ACE data available to the scientific community through the ACE Science Center and the National Oceanic and Atmospheric Administration's Space Environment Center (SEC), as summarized in Table 1. RealTime Solar Wind (RTSW) data, including measurements of energetic particles and solar wind plasma and magnetic field parameters, are broadcast continuously by ACE and tracked by NOAA, NASA, the U.S. Air Force and international partners like the Communications Research Laboratory in Japan, the Rutherford Appleton Laboratory in England, and India's Space Research Organization. The real-time solar wind data are sent to the SEC in Boulder, Colorado, where they are 


\section{Table I. ACE Data Products}

\section{The following eight instruments on the ACE spacecraft provide level 2 data products. Four of the instru- ments provide real-time solar wind products, as indicated below:}

Solar Wind Electron, Proton, and Alpha Monitor (SWEPAM) Solar-wind proton velocity, density, and temperature; ${ }^{4} \mathrm{He} / \mathrm{H}^{+}$ratio. Real-time products: Solar wind proton speed, density, and temperature.

\section{Solar Wind lon Composition Spectrometer (SWICS)}

Bulk, thermal ion speeds: $\mathrm{He}^{+2}, \mathrm{O}^{+6}, \mathrm{Mg}^{+10}$; Element ratios: $\mathrm{Ne}^{+8} / 0$, $\mathrm{Mg}^{+10} / 0$; Charge state ratios: $\mathrm{C}^{+5} / \mathrm{C}^{+6}, \mathrm{O}^{+7} / 0^{+6}$.

\section{Electron, Proton, and Alpha Monitor (EPAM)}

Spin-averaged intensities from 5 telescopes. Ions with 0.05 to $5 \mathrm{MeV}$ in 8 energy ranges. Electrons with 38 to $312 \mathrm{keV}$ in 4 energy ranges. He, CNO, and Fe in 2 energy ranges.

Real-time products: Four ion intensities, two electron intensities.

\section{Ultra Low-Energy Isotope Spectrometer (ULEIS)}

Solar/interplanetary particles. Seven species in $\sim 10$ energy ranges, 0.04-4 MeV/nuc, including ${ }^{3} \mathrm{He} /{ }^{4} \mathrm{He}$.

processed using automated routines provided and maintained by NOAA and the ACE instrument teams. These data are available on the Web within 5 minutes of measurement at http://sec.noaa.gov/ace/ACErtsw_home.html. Although not validated, and limited to a few basic parameters, the realtime solar wind data provide information on solar and interplanetary conditions upstream of Earth and can provide 30 to 60 minutes advance notice of potentially geoeffective events. The RTSW data, however, are not archived by NOAA and are not intended for use in scientific publications.

The SEC also uses ACE real-time solar wind data as a basis for issuing short-term alerts and warnings of impending geomagnetic activity. The alerts are listed online at http:// sec.noaa.gov/alerts/index.html. Other space weather products based on ACE observations are also available, including predictions of geomagnetic activity as measured by the $\mathrm{Kp}$ index that are listed at http://sec.noaa.gov/rpc/costello/index. $\mathrm{html}$. The RTSW site typically reaches more than 2000 unique users per day and peaks at over 2500 visitors during periods of high solar activity. Other data users generate additional real-time predictions, including forecasts of the Dst, $\mathrm{AL}, \mathrm{AU}$, and $\mathrm{AE}$ geomagnetic indices, magnetopause and magnetosheath locations, polar ionospheric conditions, global ionospheric electrodynamics, and geomagnetic storm and
Solar Energetic Particle lonic Charge Analyzer (SEPICA)

Solar/interplanetary particles. Eight species in $\sim 5$ energy ranges; 0.3 to $5 \mathrm{MeV} /$ nuc.

\section{Solar Isotope Spectrometer (SIS)}

Solar particles, anomalous and galactic cosmic rays. Nine species in 8 energy ranges; $\sim 6$ to $>100 \mathrm{MeV} /$ nuc.

Real-time products: $>10$ and $>30 \mathrm{MeV}$ proton intensities.

\section{Cosmic Ray Isotope Spectrometer (CRIS)}

Galactic cosmic rays: 24 species in 7 energy ranges; $\sim 60$ to $>400$

$\mathrm{MeV} / \mathrm{nuc}$.

\section{Magnetometer (MAG)}

Magnetic field vector (I6-sec resolution) in multiple coordinate systems; RMS values of underlying high-resolution measurements.

Real-time products: Magnetic field vector in GSM coordinate system.

substorm warnings. A summary of the ACE-related data links can be found at http://www.srl.caltech.edu/ACE/ASC/ related_sites.html.

Several kinds of higher-level data products are available in Hierarchical Data Format and ASCII format from the ACE Science Center, located on the Web at http://www.srl.caltech.edu/ACE/ASC/, and a subset of scientific data is also available in Common Data Format from the CDAWeb site at http://cdaweb.gsfc.nasa.gov. Browse data are made available from the ACE Science Center within 2 to 3 days of measurement and are designed for monitoring large-scale particle and field behavior and for identifying interesting time periods. These data are more accurate and more extensive than the real-time solar wind data, but are also automatically generated and are not routinely checked. The browse data are designed for exploratory purposes only, and users are requested to consult with appropriate instrument investigators before citing them.

Calibrated science data, known as level 2 data, include composition and intensity measurements of solar energetic particles, galactic cosmic rays and anomalous cosmic rays, along with solar wind and interplanetary magnetic field parameters (see Table 1). Hourly, daily, and 27-day averages of the data from eight instruments are available. Higher time resolution products include 16 -second magnetic field vectors, 
64-second solar wind parameters, and 10-second energetic electron and ion data. There is a period of about 3 months between when the level 2 data are recorded by the spacecraft and their availability at the ACE Science Center. Some level 2 data are available only from early 1998, when the spacecraft reached L1, although data for five instruments extend back to August 1997. While level 2 data are suitable for scientific study and may be freely used in publications, the ACE team requests appropriate citation to the data source.

The ACE Science Center currently fills over 3000 level 2 data requests each month for a range of solar, heliospheric, and astrophysics investigations, including magnetospheric, ionospheric, and space weather efforts. For example, Temerin and Li developed a model that predicts with surprising accuracy the geomagnetic parameter Dst that is associated the ring current using solar wind measurements and magnetic field data from ACE, as described in a 2002 Journal of Geophysical Research-Space Physics paper. When the predictions of their model were compared with measured Dst values for the first half of 2000, the average error was $7.9 \mathrm{nT}$ and the linear correlation coefficient was 0.95. A comparison between the predicted and measured values for April 2002 is shown in Figure 1.

There are also several value-added products, referred to as level 3 or contributed data, available through the ACE Science Center. These include lists of interplanetary shocks and other transient events, solar energetic particle velocity and composition spectrograms, electron pitch angle distributions, iron charge-state measurements, and various daily and monthly summary plots.

\section{Changes and Additions to ACE Data Availability}

Several new level 3 data products are now available, and others are planned for the near future. Of particular relevance to space weather studies is a merged data set of magnetic field and thermal ion moments useful to magnetohydrodynamics studies of the solar wind, heliospheric, and magnetospheric physics. This product is provided with 64-second resolution in three different coordinate systems along with spacecraft location data for use in multispacecraft studies. In addition, the Solar Wind Ion Composition Spectrometer (SWICS) team plans to deliver new solar wind abundance and charge-state ratios, as well as solar wind speeds for protons and iron, following cross-calibration with the Ulysses/SWICS instrument.

In an effort to provide improved data for solar energetic particle studies, the Electron, Proton, and Alpha Monitor team is now delivering 5-minute and 10 -second averages for ion and electron intensities, which were previously available only as 1-hour averages. The Solar Energetic Particle Ionic

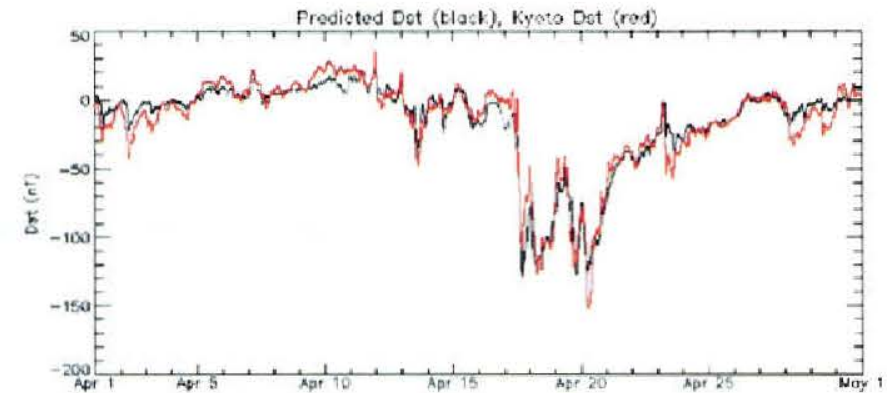

Figure 1. A comparison of the Dst values predicted by the Temerin and Li [2002] model (black line) with measured Dst values (red line) for April 2002 shows the close correlation between the predicted and measured values. Figure courtesy of Xinlin Li.

Charge Analyzer team has recently provided daily mean iron charge states and helium isotope ratios for the period from launch through December 2000. The Solar Isotope Spectrometer (SIS) and Ultra Low-Energy Isotope Spectrometer teams are reprocessing their level 2 data to incorporate improved calibrations and data analysis techniques, resulting in more accurate particle composition and intensity values. In addition, surveys of solar energetic particle and galactic cosmic ray isotopic abundances are being made available from the SIS and Cosmic Ray Isotope Spectrometer instruments.

\section{The New ACE Orbit and Fuel-Use Strategy}

The L1 orbit of the ACE spacecraft is inherently unstable, requiring four to six station-keeping maneuvers per year to keep the spacecraft bound to its orbit. In addition, the spin axis must be precessed at regular intervals to keep Earth within the beam of its high-gain antenna. During its first 4 years at L1, two additional Z-axis maneuvers were used each year to prevent the Sun-Earth-Spacecraft (SES) angle from dropping below 4.75 degrees due to the natural evolution of the Lissajous orbit (see Figure 2). With this conservative solar exclusion zone (SEZ) strategy, designed to avoid solar radio interference with the telemetry downlink, the fuel on ACE would have lasted only until 2008. In order to achieve additional lifetime, $Z$-axis maneuvers were discontinued after July 2001 , thereby allowing the Lissajous orbit to evolve, as shown in Figure 2.

As a result of this evolution, ACE will transit the solar exclusion zone with an SES angle of less than $2^{\circ}$ every three months from September 2003 through May 2005. Depending on the solar conditions, the solar radio flux may reduce the telemetry link margin to less than $3 \mathrm{~dB}$ during some of these transits, possibly interfering with the transmission of highrate science data for several days. Since the ACE solid-state recorder has a 52-hour capacity, minimal data loss is expected. 


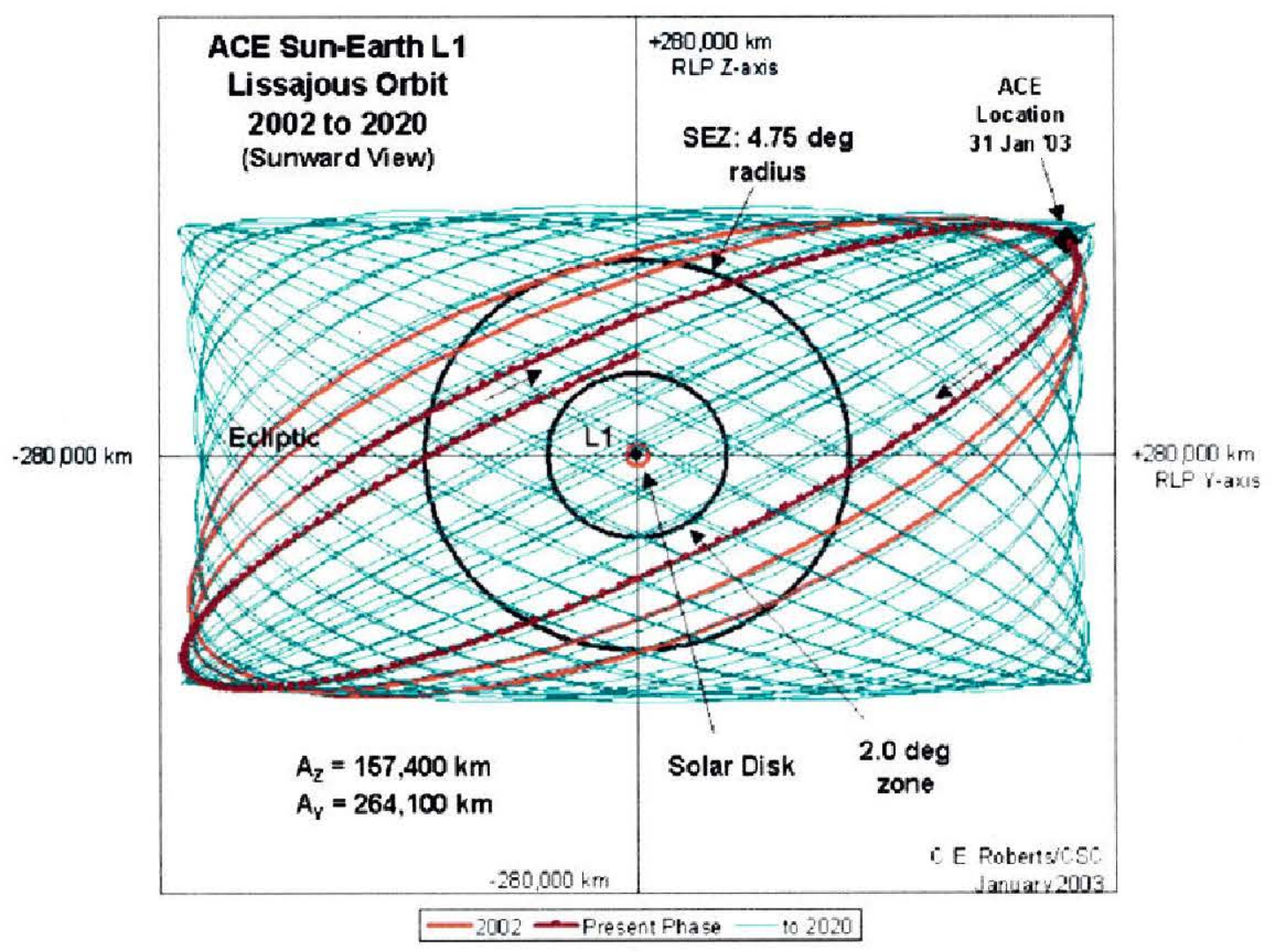

Figure 2. ACE Sun-Earth Lissajous orbit from 2002 to 2020. In order to conserve fuel, the ACE spacecraft is no longer making Z-axis maneuvers, which will allow the Lissajous orbit to evolve. (Courtesy of Craig Roberts.)

Neither NASA nor NOAA has lost any data during solar exclusion zone transits through May 2004 when the minimum SES angle was $0.2^{\circ}$ and the path of the spacecraft crossed the solar disc as viewed from Earth.

Some space weather studies could receive a benefit from the new ACE orbit because smaller SES angles may lead to improved mapping of solar wind conditions at L1 onto the magnetosphere. When the SES angle was greater than 4.75 degrees, ACE was always more than 20 Earth radii ${ }_{(\mathrm{RE})}$ from the Earth-Sun line, and was essentially never sampling solar wind directed within $10_{\mathrm{RE}}$ of the nose of the magnetosphere. During the last half of 2003, there were approximately 10 days when the solar wind sampled by ACE arrived within $1.510_{\mathrm{RE}}$ of the nose of the magnetosphere. These data, and additional data from 2004 and 2005, will be available to investigate whether forecasts of geomagnetic activity based on upstream solar wind measurements can be improved under these conditions.

As the Lissajous orbit continues to evolve, the next SEZ transits within $2^{\circ}$ occur in 2011-2012 at solar maximum. The experience during the SEZ transits in 2003-2005 will enable better estimates of the data loss duration at solar maximum. If necessary, it is possible to resume the Z-axis maneuvers prior to 2011 and avoid SES angles of less than $2^{\circ}$ during solar maximum. Each year that the Z-axis is controlled shortens the mission by 21 months. There should be enough fuel to last until 2020 if $Z$-axis maneuvers are not resumed.
The ACE spacecraft remains healthy and in good working condition, capable of continued operation and providing the high-quality data that have served the space physics community since 1998. The new orbit and fuel-use strategy will allow for the possibility of continued ACE measurements from its orbit through the next solar maximum and will also periodically place ACE closer to the Earth-Sun line.

Acknowledgments. We thank the members of the ACE Flight Operations and Flight Dynamics teams at NASA/Goddard Space Flight Center for their sustained efforts to deliver continuous, high-quality data from L1. We also acknowledge the vital contributions of Tom Garrard, who died not long before the launch of ACE. We are grateful to Xinlin Li for providing Figure 1.

A. J. Davis is manager of the ACE Science Center at the California Institute of Technology in Pasadena, California.

R. A. Mewaldt, and E. C. Stone are researchers at the Space Radiation Laboratory, California Institute of Technology, Pasadena, California.

Charles W. Smith is a research professor with the Space Science Center, University of New Hampshire, Durham, New Hampshire.

Citation: Davis, A., R. Mewaldt, E. Stone, and C. Smith (2004), Solar and Interplanetary Data From the Advanced Composition Explorer, Space Weather, 2, S07003, doi:10.1029/2004SW000062. 